ARTíCULOS DE INVESTIGACIÓN

\title{
Una invitación a pensar sobre competencias docentes en las facultades de Derecho
}

\author{
Um convite para pensar sobre competências docentes em Faculdades de Direito
}

\author{
An invitation to think about professional teaching competences \\ within the Faculties of Law
}

\author{
Lucia Irene Lapenta \\ Universidad Nacional del Centro, Argentina
}

\begin{abstract}
RESUMEN El proyecto de ALFA Tuning para América Latina propuso la implementación de competencias dentro del diseño curricular con miras a mejorar la educación universitaria. Siguiendo esta línea, en este artículo proponemos trabajar la noción de competencias en relación con el docente universitario en tanto sujeto involucrado en la relación de enseñanza-aprendizaje y formador de futuros abogados. Con este fin, ofrecemos un breve recorrido por algunos de los desafíos que entendemos como vigentes en las prácticas de enseñanza jurídica y, luego, concluimos con una reflexión sobre las potencialidades de pensar en competencias profesionales para los docentes en la Facultad de Derecho.
\end{abstract}

PALABRAS CLAVE Cultura universitaria, formación académica, formación en competencias, enseñanza del derecho.

RESUMO O projeto ALFA Tuning para a América Latina propôs a implementação de competências no desenho curricular com vistas a melhorar a formação universitária. Seguindo essa noção, propomos neste artigo trabalhar a noção de competências em relação ao professor universitário, como sujeito envolvido na relação ensino-aprendizagem e como formador de futuros advogados. Para tanto, fazemos um breve percurso por alguns dos desafios que entendemos como correntes nas práticas de ensino jurídico e, a seguir, concluímos com uma reflexão sobre as potencialidades de se pensar as competências profissionais dos professores da Faculdade de Direito.

PALAVRAS-CHAVE Cultura universitária, formação acadêmica, formação por competências, ensino do direito. 


\begin{abstract}
The ALFA Tuning Project for Latin America proposed the implementation of competences within the curricular design in the light of improving university education. Following this notion, we propose in this article to work on the notion of competences in relation to the university professor, as the subject involved in the teaching-learning relationship and trainer of future lawyers. To this end, we offer a brief overview of some of the current challenges in legal teaching practices and then, we conclude with a reflection on the potential of thinking about professional competences for professors at the Faculty of Law.
\end{abstract}

KEYWORDS University culture, academic formation, competency-based formation, law teaching.

El verdadero maestro de derecho enseñará no solo normas, sino también experiencias y valores (Goldschmidt, 1996: 20).

\title{
Introducción
}

El proyecto de ALFA Tuning para América Latina instauró una vía de reflexión sobre la posible articulación de los sistemas de educación superior en los países de América Latina, en base a la implementación de una formación por competencias dentro de los diseños curriculares de las instituciones universitarias. El proyecto Tuning surgió como producto del denominado Proceso de Bolonia, que propuso el desarrollo del Espacio Europeo de Educación Superior. A través de la Declaración de Bolonia firmada en 1999 varios países europeos se comprometieron a armonizar la arquitectura de la educación superior en Europa, trabajando en beneficio de una convergencia de contenidos y una convalidación de titulaciones dentro de un régimen transnacional común de educación superior. Al respecto, es posible ver a González Wagenaar (2006).

Como derivación, surge el ALFA Tuning América Latina como un proyecto independiente, impulsado y coordinado por universidades de distintos países latinoamericanos y europeos, en busca de reproducir la misma línea de trabajo en las instituciones de educación superior de América Latina. El objetivo principal de ambos proyectos en términos educativos es el desarrollo de la calidad, efectividad y la transparencia educativa en la misión de educar por competencias:

Es un trabajo conjunto que busca generar un espacio de reflexión sobre la educación superior y construir lenguajes y mecanismos para la comprensión recíproca de los sistemas de enseñanza superior, que faciliten los procesos de reconocimiento de carácter transnacional y transregional, en particular de titulaciones» (AAVV, 2008: 13). 
Esta formación basada en competencias surge como un nuevo enfoque para el nivel de educación superior, en superación de la tradicional formación por objetivos predominante en la década del setenta y del ochenta (Cullen, 1997).

La noción de competencia comporta una perspectiva diferente con respecto al desarrollo de contenidos u objetivos, en tanto se orienta más hacia lo que el sujeto pueda hacer, al desarrollo de habilidades y capacidades. Así, «se confía más, con las competencias, en la capacidad de respuesta ante situaciones variables e imprevisibles que en un catálogo fijado de respuestas deseables y supuestamente valiosas» (Cullen, 1997: 90). De este modo, la noción de competencia no queda reducida a una mera apropiación de conocimientos, sino por el contrario, abarca todo un conjunto de capacidades que permiten a la persona responsable convertirse en ese "ser competente" para realizar múltiples acciones. Así, la persona competente puede proyectar y evidenciar su «capacidad de resolver un problema dado dentro de un contexto específico y cambiante» (AAVV, 2008: 36).

Entonces, «la competencia, al igual que la inteligencia, no es un capacidad innata, sino que, por el contrario, es susceptible de ser desarrollada y construida a partir de las motivaciones internas de cada cual» (AAVV, 2008: 36). Las competencias pueden estar relacionadas con un área de conocimiento disciplinar específico, denominadas en este caso competencias específicas, o pueden consistir en competencias genéricas pensadas para el alumno universitario que incursione en cualquier programa de estudios.

Las competencias genéricas son atributos compartidos que pueden ser generados desde cualquier carrera y que son considerados importantes por la sociedad. Luego, las competencias específicas son aquellas que responden a cada área temática o disciplina académica. Se han identificado para el espacio de educación superior latinoamericano un total de 27 competencias genéricas (AAVV, 2008: 44/45), y así también 24 competencias específicas que atañen en particular a las carreras de Derecho o Abogacía (AAVV, 2008: 113/114). Todas estas competencias han sido pensadas en función del perfil de alumno y egresado de la carrera de Derecho o Abogacía.

En el marco del proyecto ALFA Tuning, todos los programas de estudios de educación superior son reestructurados en base a esta noción de competencias. Ahora, esta noción de competencias se elabora considerando el perfil del alumno y del futuro graduado, poseedor de la titulación universitaria respectiva. Es decir, que se proyecta el diseño curricular en base a aquellas capacidades que alcanzarán a consolidar los alumnos a lo largo de la carrera universitaria (González Ramos, 2017; Pérez Perdomo, 2016; Garay, 2019; Aedo Barrena, 2015; Palomo Vélez y Riveros Ferrada, 2015).

En este artículo proponemos retomar esta noción de competencias en una dirección diferente, es decir, no con miras a la proyección del alumnado en formación, sino más bien en relación con los docentes universitarios en tanto sujetos involucrados en la relación de enseñanza-aprendizaje dentro de las aulas y formadores activos 
de futuros abogados. Con este fin, realizaremos en primer lugar una revisión general de los desafíos actuales en la práctica de enseñanza jurídica para, finalmente, ofrecer algunas proyecciones en torno a las potencialidades de trabajar sobre competencias docentes en los espacios de formación universitaria en derecho.

\section{Desafíos actuales en las prácticas de enseñanza jurídica universitaria}

Con el objetivo de sostener nuestra propuesta, es preciso considerar someramente el panorama de la enseñanza jurídica en general. Al respecto, Cristian Giménez Corte (2003: 74) afirma que:

Las facultades argentinas se han caracterizado desde siempre, y salvando algunas excepciones, como excesivamente teóricas, memoristas y enciclopedistas [...] con una sola forma o manera de enseñar, y que es la clase magistral. A través de ella el profesor asume el rol de un maestro que transmite, solemne, una especie de verdad revelada, infalible, insusceptible de ser puesta en duda: dicta la clase. Esto genera una actitud absolutamente pasiva por parte del alumno, totalmente acrítica, y que por lo general se reduce a "tomar apuntes" de lo que dice el profesor. Este método de enseñanza responde a la tradición colonial prepositivista, y desde el punto de vista epistemológico a la dogmática jurídica, heredera de la dogmática teológica.

Así también, el propio informe emitido desde el proyecto ALFA Tuning América Latina, que reúne la experiencia de diversas universidades latinoamericanas, indica que:

La dogmática jurídica ha sido la matriz teórica dominante en la mayor parte de las facultades o cursos, con énfasis metodológico en la transmisión de contenidos teóricos por parte del docente y la absorción de estos por el estudiante. No hay una aproximación sistematizada entre la enseñanza y los procesos de aprendizaje, que, en la mayoría de las instituciones, son aun muy conservadores, fundados en una metodología de asimilación acrítica de proposiciones teóricas, o memorización de normas jurídicas. El ordenamiento jurídico, o sistema normativo es comúnmente el eje estructurante de disciplinas que se organizan como reflejo de campos jurídicos tradicionales (AAVV, 2008: 110).

Estas breves reseñas reflejan algunos de los desafíos que consideramos prioritarios en relación con las prácticas vigentes en las Facultades de Derecho. A nuestro entender, detrás de este tipo de prácticas dominantes hay dos premisas principales que permiten su reproducción en forma consistente: por un lado, se presenta una concepción del conocimiento entendido como un objeto, el cual se transmite desde el docente hacia el alumno en una vía unidireccional y, por el otro, se parte de una determinada concepción del derecho como objeto de estudio, muy cercana a una concepción dogmática o formalista. 
A continuación, desarrollamos un pequeño análisis sobre cada una de estas dos concepciones fundantes, con miras a demostrar la necesidad de su replanteamiento. Consideramos que las concepciones dominantes de conocimiento y del derecho perfilan una labor y un rol docente de tipo muy particular, y es precisamente a la luz de esta figuración docente que construimos nuestra crítica.

\section{El desarrollo de la labor docente según la concepción del conocimiento}

A nuestro entender, la concepción del conocimiento como un objeto a transmitir por el docente dentro de un espacio de educación superior resulta, en cierto modo, obsoleta. Si bien esta fue una concepción que se mantuvo vigente durante mucho tiempo, actualmente predomina la necesidad de pensar el conocimiento como un acto de construcción, desarrollado en el ida y vuelta de la relación alumno-docente y en el intercambio dentro del espacio del aula, presencial o virtual. Este cambio de concepción acompaña la transición desde un modelo educativo centrado en la enseñanza hacia un modelo centrado en el aprendizaje, donde en relación al conocimiento:

Se debe aceptar su carácter relativo y ser capaces de desenvolvernos en esa incertidumbre y complejidad. Desde esta perspectiva, frente a la concepción del conocimiento como un "constructo" cerrado, la formación universitaria ha de favorecer un aprendizaje flexible, planteando una visión del conocimiento como proceso constructivo (Fernández March, 2006: 38).

En forma concordante con este cambio de concepción sobre el conocimiento, la actividad del docente deja de pensarse como un acto mayormente expositivo que requiere de claridad y eficiencia, y se impone una revisión de las prácticas y competencias del docente universitario en términos más cercanos a la empatía y la pertinencia para aportar habilidades afines al desarrollo de una construcción colaborativa dentro del aula.

En apoyo a esta concepción constructivista del conocimiento, cabe mencionar el proceso de democratización del conocimiento propio de nuestro tiempo a raíz del cual los saberes, las ciencias y la tecnología resultan actualmente un patrimonio mucho más accesible. El acceso a las diversas fuentes de información a través de los medios digitales ha permitido un acceso al conocimiento mucho más abierto e inclusivo. En otras palabras, la sociedad del conocimiento relativiza aquellas prácticas de transmisión de información dentro del universo de tareas docentes y plantea, por el contrario, que el acto de aprender requiere mayormente de una labor de guía por parte del docente, y así también del interés, la dedicación y la voluntad de los estudiantes.

Sin embargo, por contraposición al fácil acceso a la información, también se presenta el problema de la desinformación, es decir, la circunstancia inevitable de que dentro del gran caudal de información se incluya también la "mala información". Su- 
mado a esto, no es posible dar por sentado que el acceso a la información comprenda de por sí su transformación en conocimiento propiamente dicho. La producción y sistematización de esa información en términos de conocimiento conllevan tareas adicionales que aún resta desarrollar dentro del aula, y que no quedan garantizadas con el simple acceso abierto a la información. Por esto, la sociedad del conocimiento requiere de una formación orientada al desarrollo de las capacidades del pensamiento integrador, del razonamiento creativo, de la innovación y del uso de inteligencias múltiples (Pérez Lindo, 2005).

Por último, cabe considerar un desafío adicional, como lo es la complejidad del conocimiento. En el contexto de la sociedad del conocimiento, los saberes se presentan en forma cada vez más especializada y fragmentada. Las distintas disciplinas ahondan en diferentes dimensiones de los fenómenos, explorando variantes, incorporando interpretaciones complejas e, inclusive, reconociendo un halo inevitable de imprecisión e incertidumbre. En razón de ello, los desarrollos del conocimiento, y en particular del conocimiento científico, incluyen como positivo y necesario el cruce de conocimientos a través de miradas de interdisciplinariedad y/o transdisciplinariedad, otro de los desarrollos necesarios para construir dentro del aula (Pérez Perdomo, 2009).

En definitiva, frente a estas consideraciones con respecto a la concepción del conocimiento y a las circunstancias actuales de accesibilidad informativa, creemos que la labor del docente y, consecuentemente, sus competencias profesionales, se ven cada vez más alejadas de aquellos requerimientos de claridad expositiva y de capacidad de transmisión del conocimiento. Mientras tanto, sobresalen habilidades clave como el desarrollo de la creatividad, la empatía, la capacidad de razonamiento crítico $\mathrm{y}$ aplicado, entre otras.

\section{El desarrollo de la labor docente según la concepción del derecho}

En adición a las circunstancias consideradas en el apartado previo, toda enseñanza universitaria en el ámbito del derecho incorpora una problemática que podríamos llamar «de raíz»: la inexistencia de un concepto unívoco del derecho entendido como objeto de estudio y, por ende, de una idea unívoca de enseñanza.

En este sentido, nos interesa reflexionar aquí sobre cómo las diferentes concepciones del derecho como objeto de estudio pueden llegar a imponer una determinada prioridad en términos de roles o labores docentes dentro del aula. De esta manera, y en adhesión a la perspectiva trialista integrativista del fenómeno jurídico (Goldschmidt, 1996; Ciuro Caldani, 2019), anticipamos brevemente nuestro posicionamiento al respecto: entendemos que la simplificación del derecho como objeto de estudio conlleva de manera consecuente hacia una visión simplista y restrictiva de la labor del docente universitario. Por el contrario, consideramos que la adopción de una 
concepción que aborde la complejidad del mundo jurídico permite visualizar y desarrollar el rol del docente en toda su diversidad y extensión. Con respecto a la necesidad de abordar una visión compleja e integrada del fenómeno jurídico, el doctor Ciuro Caldani afirma que «la revisión de las exclusiones del campo jurídico muestra que su apartamiento, a veces llamado "purificación", es en diversos aspecto insostenible. Se suele considerar fuera del derecho lo que a nuestro parecer posee una importancia jurídica evidente» (Ciuro Caldani, 2019: 15).

Como lo indicamos al comienzo de este texto, y según el informe de ALFA Tuning América Latina, la concepción dominante en la mayor parte de las Facultades de Derecho ha sido la dogmática jurídica o el desarrollo de un saber jurídico formalista. Dentro de la concepción formalista, el derecho se concibe en términos generales como un conjunto de normas emanadas de los órganos creados al efecto. Cabe mencionar en esta línea a la escuela de la exégesis, la jurisprudencia de conceptos y la jurisprudencia analítica, desarrollos influyentes del siglo XX que se caracterizaron por una concepción puramente normativa y estatal del derecho.

Cabe considerar entonces cómo queda traducida la enseñanza jurídica en el marco de esta concepción del objeto derecho. En principio, entendemos que la finalidad preponderante en términos de enseñanza-aprendizaje es lograr que los alumnos conozcan el ordenamiento normativo y su contenido. Concordantemente, las actividades de práctica y evaluación dirigidas a los alumnos se vincularán en su mayor parte a la reproducción de su contenido o la aplicación del texto normativo.

En este marco, el perfil docente resulta altamente compatible con el perfil del profesional del derecho, en tanto conocedor y aplicador del sistema de normas (Martín Böhmer, 1999; Rogelio Pérez Perdomo, 2009; María Inés Bergoglio, 2006). A su vez, las competencias del docente giran en relación con la capacidad de explicación y transmisión del conocimiento sobre el derecho formalizado, es decir, que la actividad docente se convierte mayormente en una transmisión de contenido normativo $y$, en menor medida, en el desarrollo de habilidades relativas a su aplicación, interpretación, etcétera.

En un escenario diferente, las perspectivas críticas del derecho permiten incorporar dentro de la enseñanza jurídica un énfasis en los elementos discursivos, ideológicos y pragmáticos que integran el fenómeno jurídico. Dentro de estas perspectivas críticas, cabe mencionar la corriente del realismo norteamericano y escandinavo, la Escuela del Derecho Libre, los Critical Legal Studies (CLS) y las perspectivas críticas del derecho en Latinoamérica. En términos generales, las diferentes propuestas de estas perspectivas se fundan en una fuerte crítica a la razón jurídica teórica y buscan visibilizar el fenómeno jurídico como una práctica social instrumental o discursiva.

Entonces, en clave de formación educativa se incorporan dentro del aula las tareas de análisis de los aspectos sociales, políticos y culturales que circundan al derecho, más allá del desarrollo de los contenidos conceptuales (Mosquera Schvartz, 2015). A 
su vez, como derivación de esta peculiar concepción del derecho, la enseñanza jurídica incorpora necesariamente tareas como el debate y la discusión crítica, el análisis de discurso y de relaciones de poder, en contraste con aquella enseñanza centrada exclusivamente en la interpretación normativa.

Desde las perspectivas críticas, el perfil docente parece acercase también al perfil profesional de derecho, aunque desde un costado diferente, es decir, se extiende hacia aquellos aspectos de compromiso social, de responsabilidad colectiva, de análisis de los intereses en conflicto, más allá de la práctica de aplicación, desarrollo y análisis de lo normativo en el marco del litigio judicial. A nuestro entender, bajo esta concepción del derecho las competencias del docente se extienden para incorporar habilidades clave como la oratoria, la argumentación, el análisis crítico y el comparado. En concreto, gran parte de la labor docente se convierte en un cruzamiento de aspectos jurídicos con conceptos provenientes de teoría social, filosofía del derecho, teoría del Estado, entre otras disciplinas. De esta manera, se traducen los contenidos del sistema jurídico en términos de alternativas de transformación y cambio social.

Por otro lado, si tomamos como referencia el marco de una concepción analítica del derecho, las tareas de enseñanza también se extienden más allá del conocimiento sobre el ordenamiento normativo hacia el desarrollo de habilidades de argumentación, de estrategia jurídica y de métodos de solución de conflictos. Esta concepción se ubica en cercanía a las posturas de la argumentación jurídica sostenidas, por ejemplo, por los doctores Carlos Nino, Manuel Atienza Rodríguez y Martín Böhmer.

En relación con el contenido de las prácticas de enseñanza-aprendizaje, esta concepción plantea como base fundamental la comprensión de un sistema de reglas, principios, directrices y sus interrelaciones. En términos analíticos, entonces, no se abordan exclusivamente las tareas de conocimiento y aplicación, sino también el desarrollo de un razonamiento práctico, de la ponderación y la interpretación en relación con ese sistema de reglas y principios que constituye el derecho. De este modo, se incorporan también consideraciones sobre la moral y la política, entendidas como fuentes de cuestionamiento muy cercanas a lo jurídico y fuentes de justificación racional frente a la toma de decisiones.

A nuestro entender, el perfil docente en este marco analítico resulta afín a la dimensión profesional de la abogacía, pero así también a la labor del investigador en derecho en tanto se indaga en materia de fines, justificación, validación y racionalidades dentro del derecho. Luego, en términos de competencias docentes orientadas bajo esta concepción del derecho, entendemos que se destacan las habilidades prácticas, principalmente las de razonamiento, argumentación y análisis. Las actividades de enseñanza-aprendizaje desarrolladas en este contexto se traducen en su mayor parte en el análisis de la estructura analítica operante dentro del sistema jurídico y sus interconexiones en términos de interpretación, de ponderación, de resolución de conflictos y de modificaciones al régimen estatuido. 
Por último, proponemos analizar la concepción del tridimensionalismo integrativista (o teoría trialista del mundo jurídico), en la que el fenómeno jurídico se concibe como una complejidad desarrollada en una tridimensionalidad de hecho, norma y valor. Aquí, entendemos que la enseñanza del derecho incorpora una amplitud de prácticas y matices concordantes con la multidimensionalidad integradora que se atribuye al propio mundo jurídico. En este sentido, por ejemplo, los contenidos de la dogmática jurídica no resultan desestimados, sino que se incorporan como una visión parcial del objeto jurídico complejo.

Desde la perspectiva trialista, la enseñanza sobre el mundo jurídico se integra con matices de filosofía del derecho, de teoría social, de historia del derecho y de axiología, entre otros. En términos generales, las actividades de enseñanza tienen por finalidad última lograr un aprendizaje integrado con conocimientos, habilidades y estrategias para la toma de decisiones, para identificar y resolver conflictos, para apreciar el alcance de esos conflictos en términos de fraccionamiento y desfraccionamiento dentro de una complejidad mayor.

A nuestro parecer, el trialismo propone como labor docente el desarrollo de prácticas de enseñanza-aprendizaje a través de un caos constructivo, que integra el orden y también el desorden, que aporta riqueza en la perspectiva del fenómeno jurídico en base al desarrollo estratégico de un razonamiento teórico-práctico. Dentro de este caos constructivo se integran las prácticas de análisis de casos, de estudio del funcionamiento de normas y ordenamientos, y de valoración en términos de axiología y dikelogía. En palabras del doctor Ciuro Caldani (2019: 16):

Los alumnos que ingresan a la Facultad de Derecho vienen a aprender una profesión, no sólo la lógica, las abstracciones de referencias valorativas a priori o los hechos. La ciencia ha de referirse a los despliegues más plenos posibles de la vida y no ésta a los deseos de los científicos enclaustrados y razonadores y «moralistas» abstractos o practicones que la recortan caprichosamente.

De esta manera, la perspectiva trialista permite ampliar la visión del fenómeno jurídico en sus diferentes dimensiones y, para ello, amplía el rango de movilidad del aprendiz frente un objeto de estudio multidimensional (Ciuro Caldani, 2005a). Aprender sobre el derecho se transforma, a partir de las categorías propias de la teoría trialista del mundo jurídico, en un juego que se desarrolla desde la dimensión social hacia lo construido y viceversa; desde lo individual hacia lo colectivo y viceversa; desde una perspectiva macro hacia una mirada micro y viceversa; desde los fraccionamientos hacia los desfraccionamientos y viceversa. Todo este recorrido, en sus especificidades y en su dinámica, será lo que se reconstruya (y coconstruya) dentro del aula para lograr apreciar el mundo jurídico en su complejidad pura.

Por todo esto, entendemos que el perfil docente dentro de esta concepción trialista resulta afín a la dimensión profesional de la abogacía, pero así también a la labor de 
investigación en derecho, a la actividad del operador del derecho y a la visión del filósofo del derecho. En última instancia, el perfil docente resulta también semejante a la condición del ser humano (o repartidor), considerando que desde la teoría trialista se concibe el derecho como «una perspectiva de la vida humana toda (de cierto modo de la vida toda), que nos incumbe de diversas maneras a todos como individuos y partes del complejo humano» (Ciuro Caldani, 2019: 16).

En concordancia con esto, creemos que las competencias del docente dentro de esta perspectiva se multiplican exponencialmente en comparación con los perfiles docentes analizados en el resto de las concepciones aquí desarrolladas. Luego, en cuanto a la actividad docente, la misma se convierte en el desarrollo de una percepción tridimensional integrativista con respecto al fenómeno jurídico, abarcativa de los horizontes y las especificidades particulares del mundo jurídico.

Hasta aquí, hemos intentado reflejar el modo en que las concepciones del conocimiento y del derecho mismo (que radican en el trasfondo del diseño curricular, o que sostiene el propio docente) van direccionando la labor docente en el aula, su perfil y sus requerimientos en términos de competencias profesionales. A continuación, y a modo de cierre, ofrecemos algunas reflexiones en torno a las potencialidades de pensar en términos de competencias docentes dentro de una Facultad de Derecho.

\section{Potencialidades de pensar en términos de competencias docentes}

A nuestro entender, resulta positivo - y más aún necesario- pensar en términos de competencias docentes, lo cual implica trabajar desde la institución educativa en una proyección, no solo del perfil del alumnado, sino también del cuerpo docente en tanto grupo de formadores activos. No incluiremos en esta instancia la identificación detallada de las competencias docentes que consideramos necesarias, indagación que sería altamente provechosa pero excedería el marco de este texto. Sin embargo, sí nos interesa ofrecer algunas reflexiones en torno a su potencialidad en el marco de la educación superior.

A grandes rasgos, la labor del docente universitario comprende el desarrollo de tareas de enseñanza-aprendizaje, de mecanismos de evaluación, de propuestas de aplicación práctica, la selección de herramientas, la definición de métodos pedagógicos y la recopilación de materiales de estudio, entre otros. Los procesos más tradicionales de enseñanza en el ámbito del derecho se han expandido en base a ciertas prácticas que, de acuerdo con todos los argumentos que hemos desarrollado, podrían ser consideradas actualmente como deficientes. Así, por ejemplo, el exagerado énfasis en la asimilación y memorización de contenidos conceptuales, el abuso de aquella metodología conocida como "clase magistral", la segmentación marcada entre ramas y la separación de "lo jurídico" del resto de las disciplinas sociales.

A lo largo de este artículo, hemos intentado demostrar cómo influyen la concep- 
ción del conocimiento y del derecho en el sostenimiento de estas prácticas arraigadas. Con miras a un cambio trabajado desde lo institucional, consideramos que pensar en términos de competencias docentes resulta una alternativa que configura un gran aporte. Para esto, es preciso cuestionar de manera estratégica desde la institución qué concebimos como conocimiento; qué concebimos como derecho; qué perfil docente buscamos en consecuencia; y qué competencias profesionales podemos considerar como relevantes o de desarrollo prioritario en los docentes universitarios.

De acuerdo con nuestra propuesta, consideramos que el docente debe trabajar en sus propias competencias para contribuir luego a formar personas competentes a través de su labor diaria. Ahora, ¿cómo podemos proyectar ese perfil docente y definir las competencias esperadas? En principio, entendemos que resulta coherente y preciso exigir del docente las mismas cualidades que visualizamos para el perfil profesional de los abogados, como el conocimiento sobre las estructuras jurídicas, competencias de comprensión sobre las problemáticas de carácter jurídico, competencias de sensibilidad frente a los criterios de lo justo y del bien común, con un sentido de responsabilidad social y humanismo. Sin embargo, la visión de competencias no termina aquí.

Resta considerar las competencias derivadas de los perfiles de investigador, operador y filósofo del derecho. Pero, además, resta considerar que el concepto de "competencia" en sí mismo propone hacer mayor énfasis en el desarrollo de habilidades y destrezas para enfrentar cambios y situaciones inciertas por sobre la adquisición de conocimiento o el logro de objetivos preestablecidos. En concreto, el concepto de competencia supone cierta aptitud del docente para desarrollar actividades en base a una integración compleja de conocimientos, habilidades, destrezas, aptitudes y actitudes. De este modo, tanto las tareas de enseñanza-aprendizaje como las de evaluación integrarán en sí mismas conocimientos, habilidades, destrezas, aptitudes y actitudes. Así, por ejemplo, podrá evaluarse una habilidad operativa en relación con una aplicación de normativa, una habilidad organizativa para desarrollar tareas grupales dentro de la clase, una destreza estratégica o resolutiva para argumentar la defensa o fundar la resolución dentro de un caso hipotético.

En particular, consideramos que la tarea del docente en el sistema de enseñanza universitaria de nuestro tiempo debe dirigirse a potenciar aptitudes como la capacidad crítica y creativa, actitudes como la autonomía de los alumnos para generar sus propias posturas o planteamientos, habilidades para trabajar sobre diversas fuentes de material informativo o diversas perspectivas, y destrezas para aprehender en función de procesos de aciertos y errores.

En suma, el docente precisa desarrollar aquellas competencias que le permitan construir dentro del aula un espacio de aprendizaje significativo y aplicado, compartir con los alumnos aquellas herramientas necesarias que les permitan desempeñarse por sí mismos en el ámbito universitario y dentro del mundo jurídico, gestionando 
sus propios futuros procesos de aprendizaje (Anijovich y Cappelletti, 2017; Baraona, Cadiz y Villanueva, 2015).

En último lugar, nos interesa destacar una conexión muy especial que encontramos entre nuestra propuesta de desarrollar competencias docentes y el marco de aprendizaje que ofrece la concepción iusfilosófica trialista del mundo jurídico.

Por un lado, la propuesta de formación por competencias y la perspectiva trialista incluyen una cuota ínsita de incertidumbre. Así, adquirir competencias implica adquirir un saber hacer para poder actuar frente a la situación que se presente:

Formar competencias es formar sujetos capaces de definir fines y medios, alternativas fundadas y estrategias diversas, capacidad, en última instancia, de evaluar desempeños a la luz de las posibilidades que vienen de las competencias adquiridas, y no de la demanda específica de determinados desempeños (Cullen, 1997: 92).

De este modo, todo desarrollo de competencias implica trabajar sobre diversos tipos de racionalidades que van más allá de la racionalidad teórica e incluso habilidades que escapan a la órbita de la racionalidad misma.

Por otro lado, la formación por competencias y el trialismo incorporan por igual una consideración sobre la complejidad del mundo (Ayala Rojas, 2013). La concepción trialista postula que como consecuencia de la complejidad propia del derecho como objeto, toda formación jurídica requiere ser trabajada desde una visión integral del espacio de educación y los sujetos que intervienen en los procesos de enseñanzaaprendizaje. En términos de competencias, esta visión integral se logra trabajando, por ejemplo, en el desarrollo de competencias transversales (a los conocimientos disciplinares), competencias interpersonales (relativas a la relación entre personas), de habilidades sociales, de comunicación, de argumentación y de habilidades técnicas, que avancen más allá del exclusivo conocimiento conceptual.

\section{Conclusión}

A lo largo de este artículo hemos intentado demostrar cómo el «espacio real de conducción» en la educación superior en derecho se encuentra condicionado, a fin de cuentas, por la concepción del conocimiento, la concepción del derecho y por la figura del docente en mayor medida que por el perfil de alumno o graduado que podamos proyectar en forma abstracta.

Consideramos que incluir la reflexión sobre competencias docentes dentro de las Facultades de Derecho puede resultar una alternativa muy prometedora en términos de fortalecimiento de la educación superior y de la propia cultura universitaria. Después de todo, la universidad es aquella institución en la que se ofrece y se genera un espacio de coconstrucción. En palabras de Goldschmidt (1996: 106): 
He aquí precisamente la diferencia abismal entre colegios y universidades. Las universidades son centros de investigación; y los profesores universitarios no se limitan a transmitir conocimientos sino que se esfuerzan en contagiar a los discípulos espíritu y técnicas de la investigación.

Por esto, entendemos que una vía de trabajo aún pendiente es considerar la noción de compentencias en relación con los docentes como "fuerza de contagio" activa de la propia institución educativa para poder luego coconstruir esos procesos de formación en competencias entre (y no sobre) alumnos y docentes.

\section{Referencias}

Aedo Barrena, Cristián (2015). "Formación por competencias y enseñanza del Derecho”. Revista Pedagogía Universitaria y Didáctica del Derecho, 1 (1): 104-113. Disponible en bit.ly/2Wex2jH.

Anijovich, Rebeca y Graciela Cappelletti (2017). "La planificación de la enseñanza en la formación de profesores en ciencias jurídicas". Academia Revista sobre Enseñanza del Derecho, 30: 87-128. Disponible en bit.ly/2UQ4tZo.

Ayala Rojas, Dora Esther (2013). "Aportes trialistas para un diseño curricular integrado". Academia Revista sobre Enseñanza del Derecho, 22: 181-196. Disponible en bit.ly/342N7wX.

AAVV (2008). Tuning América Latina. Reflexiones y perspectivas de la Educación Superior en America Latina. Informe final Proyecto Tuning América Latina 20042007. 1. ${ }^{a}$ ed. España: Universidad de Deusto.

BARAONA, Jorge, Janet Cádiz y Olga Villanueva (2015). “Thinking like a lawyer: experiencias didácticas en el aula para fortalecer la formación de un abogado". Revista Pedagogía Universitaria y Didáctica del Derecho, 2 (2): 44-54. Disponible en bit. ly/37OUOrL.

Bergoglio, María Inés (2006). "Las facultades de derecho argentinas entre las tradiciones y los esfuerzos de cambio”. En Rogelio Pérez Perdomo (editor), La formación jurídica en América Latina: Tensiones e innovaciones en tiempos de la globalización (pp. 101-142). Bogotá: Universidad Externado de Colombia.

BöHmer, Martín (1999). La enseñanza del derecho y el ejercicio de la abogacía. Barcelona: Gedisa.

Ciuro Caldani, Miguel Ángel (2005a). “Derecho de la Educación”. Academia Revista sobre Enseñanza del Derecho, 5: 135-154. Disponible en bit.ly/2UPm9nx.

-. (2005b). "Reflexiones sobre calidad educativa, política, gestión y formación". Revista Investigación y Docencia, 38: 85-95. Disponible en bit.ly/2UP5zUR.

-. (2019). Una teoría trialista del mundo jurídico. 1. ${ }^{\mathrm{a}}$ ed. Rosario: Facultad de Derecho de la Universidad Nacional de Rosario. 
Cullen, Carlos (1997). Crítica de las razones de educar. Temas de la filosofía de la educación. 1. ${ }^{\mathrm{a}}$ ed. Buenos Aires: Paidós.

Fernández March, Amparo (2006). "Metodologías activas para la formación de competencias". Revista Educatio siglo XXI, 24:35-56. Disponible en bit.ly/36WkX05.

GaraY, Tomás Eduardo (2019). "Formación por competencias y prácticas pedagógicas: reflexiones en torno al rediseño curricular de la carrera de derecho de la Universidad de Atacama”. Revista Pedagogía Universitaria y Didáctica del Derecho, 6 (1), 79-100. Disponible en bit.ly/340CMle.

Giménez CoRTe, Cristián (2003). “Enseñar derecho no es solo conocer la ley”. Revista Duc in Altum, 4: 73-80. Disponible en bit.ly/3gA2Okg.

Goldschmidt, Werner (1996). Introducción filosófica al derecho. La teoría trialista del mundo jurídico y sus horizontes. $6 .^{\mathrm{a}} \mathrm{ed}$. Buenos aires: Depalma.

GonzÁLEz, Julia y Robert Wagenaar (2006). Tuning Educational Structures in Europe. Informe final. Proyecto piloto. Fase 2: La contribución de las Universidades al Procesos de Bolonia. Bilbao: Deusto.

González Ramos, Rosalba Georgina (2017). "Exploración de planes de estudios de escuelas de Derecho y el desafío de cambio al enfoque por competencias". Academia Revista sobre Enseñanza del Derecho, 30: 129-152. Disponible en bit. ly/3nNgGKA

Mosquera Schvartz, David Manuel (2015). "Problemáticas epistemológicas en la planificación de la enseñanza del derecho”. Academia, Revista sobre Enseñanza del Derecho, 25: 203-2016. Disponible en bit.ly/3pS1AoY.

PAlomo Vélez, Rodrigo y Carolina Riveros Ferrada (2015). "La transformación curricular en derecho: Consideraciones desde la experiencia docente y de gestión académica". Revista Pedagogía Universitaria y Didáctica del Derecho, 2 (2): 55-75. Disponible en bit.ly/39ZUmte.

PÉrez Lindo, Augusto (2005). Gestión del conocimiento. Un nuevo enfoque aplicable a las organizaciones y la universidad. Buenos Aires: Grupo Norma.

Pérez Perdomo, Rogelio (2009). "Desafíos de la educación jurídica latinoamericana en tiempos de globalización”. La educación legal y la garantía de los derechos en América Latina, El Otro Derecho, 38: 11-28. Disponible en bit.ly/34EGd14.

-. (2016). “Reformar la educación jurídica ¿Tarea para Sísifo?". Revista Pedagogía Universitaria y Didáctica del Derecho, 3 (1): 3-27. Disponible en bit.ly/3mdRNqh.

\section{Sobre la autora}

Lucia Irene Lapenta es abogada, magíster en Sociología Jurídica por el International Institute for the Sociology of Law (IISL) y doctora en Sociología Jurídica por la Universidad del País Vasco (UPV/EHU). Además, es docente en la Facultad de Derecho de Azul de la Universidad Nacional del Centro de la Provincia de Buenos 
Aires, Argentina, integrante del Centro Interdisciplinario de Estudios Políticos, Sociales y Jurídicos (CIEP-UNICEN) y del Instituto de Estudios Jurídicos y Sociales (IEJUS-UNICEN). Su correo electrónico es luciailapenta@gmail.com. (D) https://orcid.org/oooo-0oo1-8646-8349. 
